\title{
Proposal for a Clinical Approach to Geriatric Patients with Anchor Need on Implant for Removable Denture: New Technique
}

\author{
Saverio Ceraulo *, Alessandro Leonida, Dorina Lauritano ${ }^{\circledR}$, Alessandro Baldoni, \\ Salvatore Longoni, Marco Baldoni and Gianluigi Caccianiga ${ }^{(1)}$ \\ Dental School, Department of Medicine and Surgery, University of Milano-Bicocca, 20126 Milan, Italy; \\ alessandro.leonida@unimib.it (A.L.); dorina.lauritano@unimib.it (D.L.); alessandro.baldoni@unimib.it (A.B.); \\ salvatore.longoni@unimib.it (S.L.); marco.baldoni@unimib.it (M.B.); gianluigi.caccianiga@unimib.it (G.C.) \\ * Correspondence: saverio.ceraulo@unimib.it; Tel.: +39-393-792-9358
}

Received: 14 June 2020; Accepted: 23 July 2020; Published: 3 August 2020

\begin{abstract}
A method is proposed using a silicone tube to allow the restoration of incongruous prostheses due to the retention of removable prostheses anchored to implants by ball-attachment, also in medical facilities Health Care Residence (RSA) where the equipment characteristic of dental clinics is lacking. One hundred and thirty-seven patients belonging to the Health Care Residence of the Monza and Brianza area were analyzed. Of these, 13 required retention replacement for ball attachment of total lower prostheses to implant anchorage. The new retention procedure was carried out with the patients bedridden according to the methodology of the present work. All 13 patients were perfectly rehabilitated in the Health Care Residence offices without discomfort and pain. The method presented is easily repeatable, risk-free and can also be carried out in structures not dedicated to dentistry, saving economic resources and inconveniences for patients who are not cooperative.
\end{abstract}

Keywords: implants; ball attachment; overdenture

\section{Introduction}

Geriatric dentistry or odontogeriatrics is that branch of dentistry that studies the oral cavity in elderly or elderly patients who are self-sufficient and not self-sufficient. Through interdisciplinary collaboration the geriatric patient is evaluated both for generic medical conditions (diabetes, heart disease, metabolic alterations, and other systemic and neurological pathologies) and for psychological conditions (anxiety, depression, economic situations) in order to improve nutritional and pisological evaluation and aesthetic. A study on the relationship between dementia and nutrition highlights how, in all stages of dementia, proper oral nutrition with high-quality foods that the patient enjoys in a pleasant environment with nursing support is essential to avoid malnutrition. Oral food supplements are recommended when food does not meet nutritional requirements. The study also clarifies that the benefits between food and nutrient supplements on cognitive abilities are not demonstrated, however [1]. In Italy, after the law 833 of 1978 that sanctioned the birth of the national health system, nursing homes (Health Care Residence) were born, as non-hospital structures, but still with a health footprint, which host people who are not self-sufficient for a variable period from a few weeks to an indefinite period, which cannot be cared for at home, and which require specific medical care from more than one specialist and comprehensive health care. The patient/host of the Health Care Residencies is then followed by highly specialized personnel with the aim of improving the quality of life of the patient. Often, the elderly guest of the Health Care Residence structure needs specific care and specific aids to be able to have a better life, despite the fact that even from a psychological point of view 
the problems persist in some subjects. Some researchers have related apathy and fragility in elderly subjects by demonstrating how these conditions potentially share physiological mechanisms and are closely associated [2]. Guests of Health Care Residence aged care facilities often have poor health and oral hygiene, periodontal disease, prosthetic problems, and overall oral neglect with consequent difficulty in feeding. Some residents have a feeding by tube, while others have an oral diet; the latter can be reintroduced in patients with feeding with tube also due to the overall and oral health conditions. A retrospective study conducted on patients fed with a tube-based protocol, an interdisciplinary team, showed how in some subjects oral feeding was successfully reintroduced in inpatients in nursing homes previously fed with a tube [3]. A cross-sectional study related the loss of teeth and masticatory capacity and nutritional indexes in the elderly, demonstrating a greater variation in body index and albumin levels in women with low masticatory capacity [4]. The maintenance of oral hygiene and the use of new techniques and protocols made it possible to reduce complications, such as mucositis and peri-implantitis around the implants, due to the accumulation of bacterial residues maintaining the health of the oral cavity and the osseointegration of the implants. Caccianiga $G$ and others have shown how the bactericidal action of the laser associated with hydrogen peroxide has improved peri-implant tissues [5-7]. The overdenture on implants with ball-attachment represents an excellent solution for all those patients who cannot face more complex rehabilitations. Čelebić A et al. have shown at 2 years follow-up and using three different types of prosthetic rehabilitation treatment that the overdenture retained with mandibular MDI (mini dental implants) can be considered as a preferred treatment by patients [8]. The insertion and removal of the overdenture on boll-attachment in the oral cavity involves the wear of the retentive means less resistant to wear with the consequent reduction of the retention of the prosthesis in the oral cavity; furthermore, other prosthetic complications can occur, as demonstrated in one of Karabuda C et al. [9]. Abou-Ayash S. et al., in a clinical study with a 5-year follow-up, showed how retention of Teflon (female portion of the retention) decreases significantly over time, and good retention at initial values is restored by changing the Teflon, while, in the ball attack (male portion of retention), the retention forces depend on the position of the implant [10].

\section{Aim}

The variation of the vertical occlusal dimension of the geriatric patient is constantly evolving due to wear of the resin of the teeth [11], and, when this is very low and the thickness for the insertion of the metal containers is very reduced, the tefon/o-ring retention means must be positioned directly in the resin. Alternatively, to position metal containers, the resin must be increased in the lingual portion of the lower prosthesis with consequent very annoying thickening for the patient. The replacement of the Teflon/o-rings presents the risk that the resin used to fix the retentive means it can go into the undercut of the retentive ball, causing considerable inconveniences. The aim of the work was to reduce the risk that the resin may end up below the ball-attachment frame and the refining time.

\section{Materials and Methods}

From February 2017 to July 2019, 137 dental visits were performed for prosthetic problems on patients in nursing homes in Lombardy. Of these 137 male and female patients, 29 are self-sufficient ambulatory, 76 self-sufficient, not self-sufficient, 23 are not self-sufficient and do not walk, 9 are bedridden, and the average age of all patients is 87.6 years. Only 13 patients ( 10 non-ambulatory self-sufficient, 2 self-sufficient walking, 1 bedridden) needed to replace the two o-ring/teflon on the ball-attachment of the lower prosthesis. The aim of the following work was to improve the grip of the lower prosthesis with retention on implants and ball-attachment, reduce the risk that the resin may end up in the undercut of the retention ball-attachment, and reduce processing and finishing times so as to not create the slightest inconvenience to the patient. In order to operate on patients who were not cooperating, we took advantage of the help of a social health worker who, together with the dentist, participated for the entire duration of the work. The new technique involves the use of pink acrylic autopiolimerizing resinsoft Flexacryl and hard Paladur, Teflon o-rings of Rhein normal 
measure, a small translucent hose in Versilic Silicone Saint Gobain diameter 5-7 mm, the laboratory motor for finishing, cutters, and polishers. All patients were included in an oral hygiene protocol.

Each patient was treated according to the following protocol:

1. The first phase for the replacement of Teflons without metal containers consists in removing the old Teflons and inserting the soft resin to allow a better adaptation of the prosthesis in occlusion (Figures 1, 3 and 5).

2. After 7 days, the Teflon/o-ring is replaced. After preparing the housing for the Teflon on the mobile prosthesis and evaluating the necessary height, insert the small silicone tube cut at the right height in the ball-attachment. Then, the Teflon is inserted. The silicone tube adheres superiorly to the Teflon inferiorly to the gingiva protecting the entire retentive attachment (Figures 2, 4, 6 and 7). After mixing the acrylic resin and inserting it in the right quantity (filling the previously prepared cavity $50 \%$ ), the prosthesis is fitted into the oral cavity, causing the patient to close in full intercuspidation and waiting for polymerization (Figures 8-10). Once the polymerization has taken place, the mobile prosthesis can be removed very easily as the acrylic resin will be present only around the Teflon and will have filled all the walls of the cavity previously prepared leaving them smooth; there may be small smears of resin that will easily be removed in a short time with the aid of rubber pads (Figure 11).

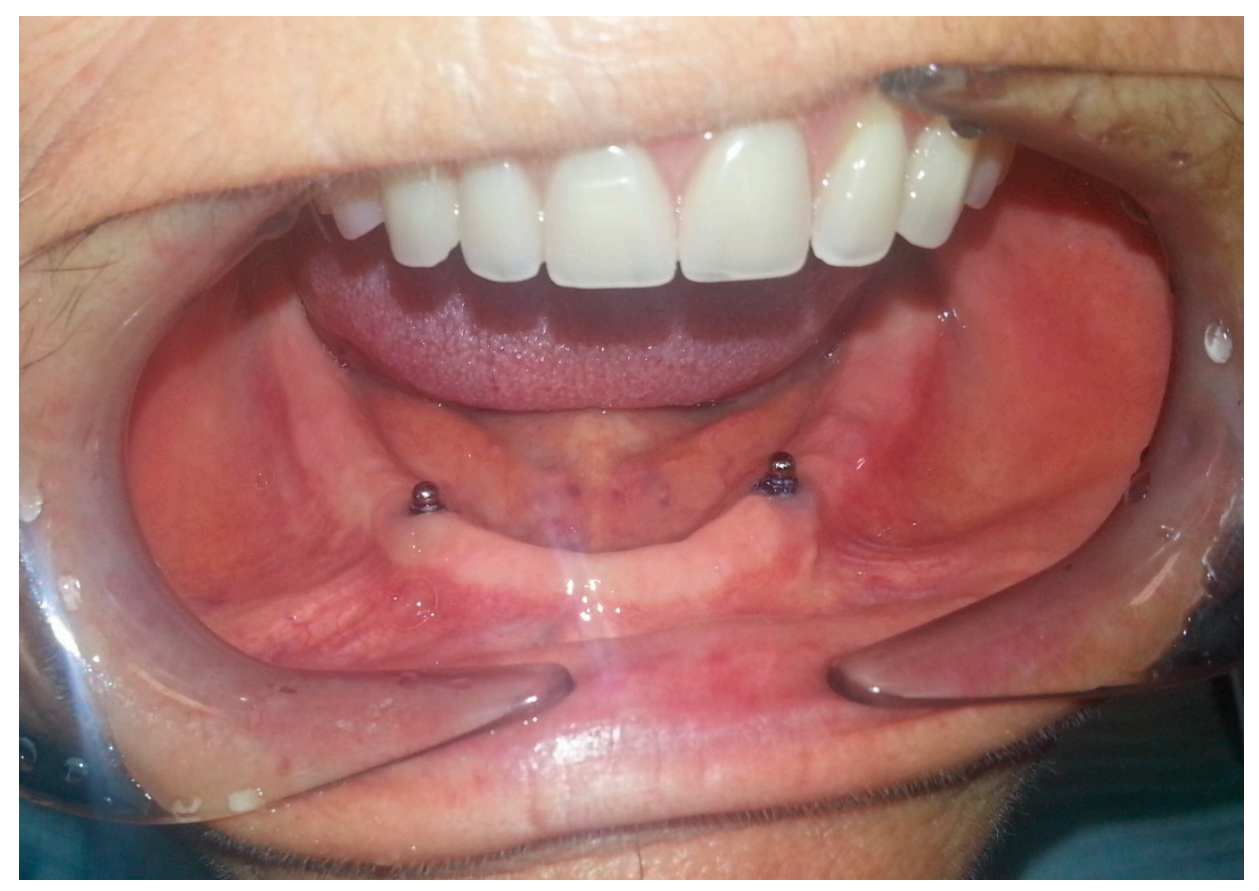

Figure 1. Patient, 77 years, guest at Health Care Residence (RSA). Lower ball attachments. 


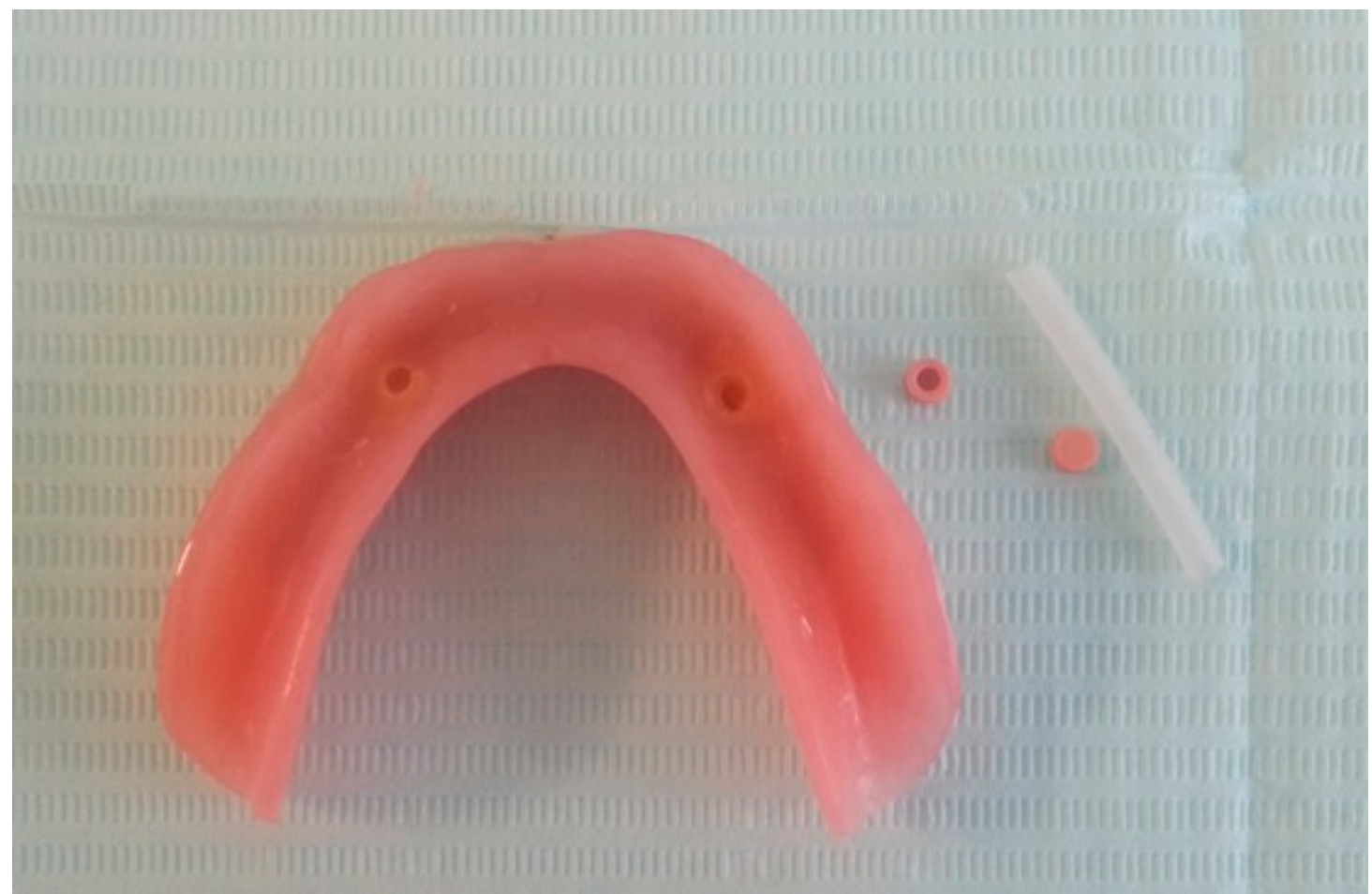

Figure 2. Silicone and Teflon tube to be inserted instead of the soft flexacril.

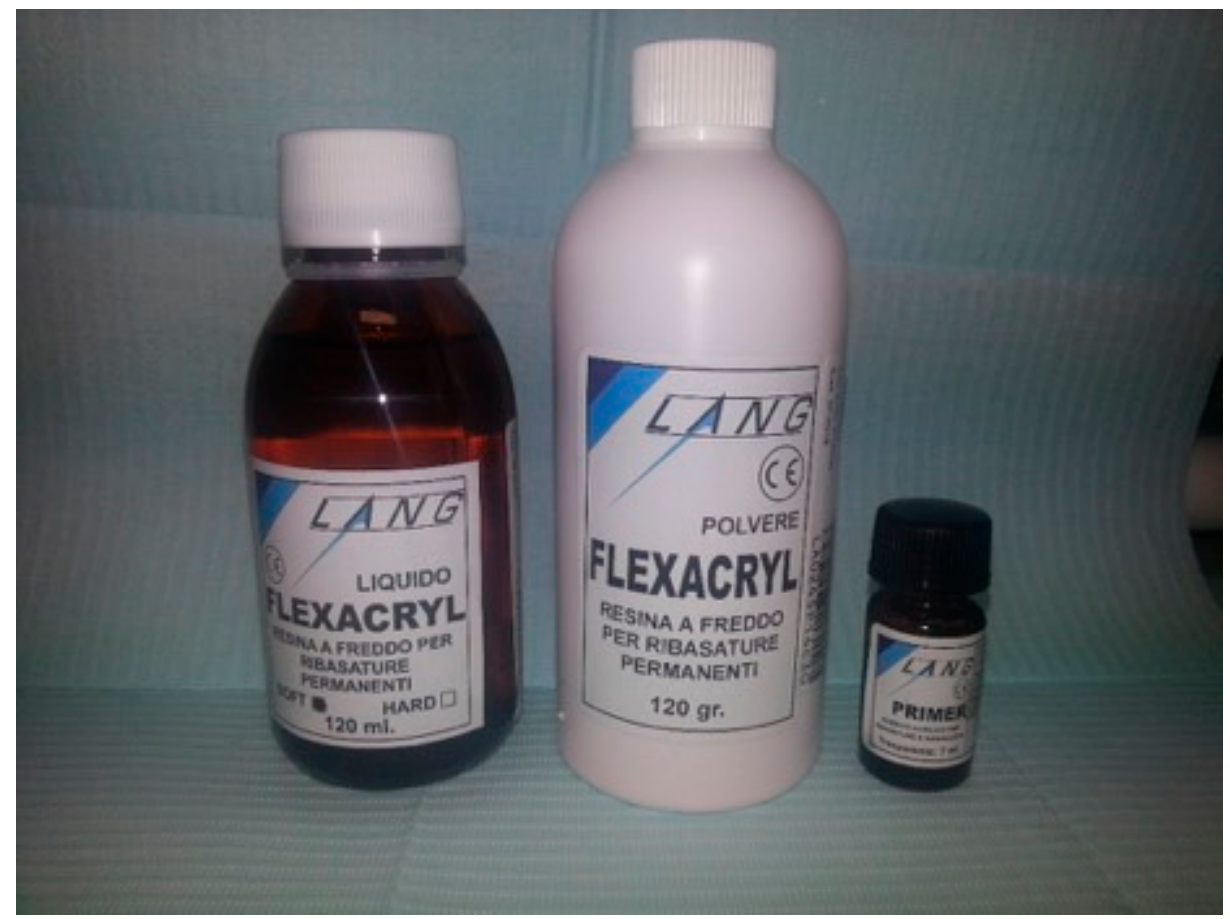

Figure 3. Soft acrylic resin for Teflon housing relining. 


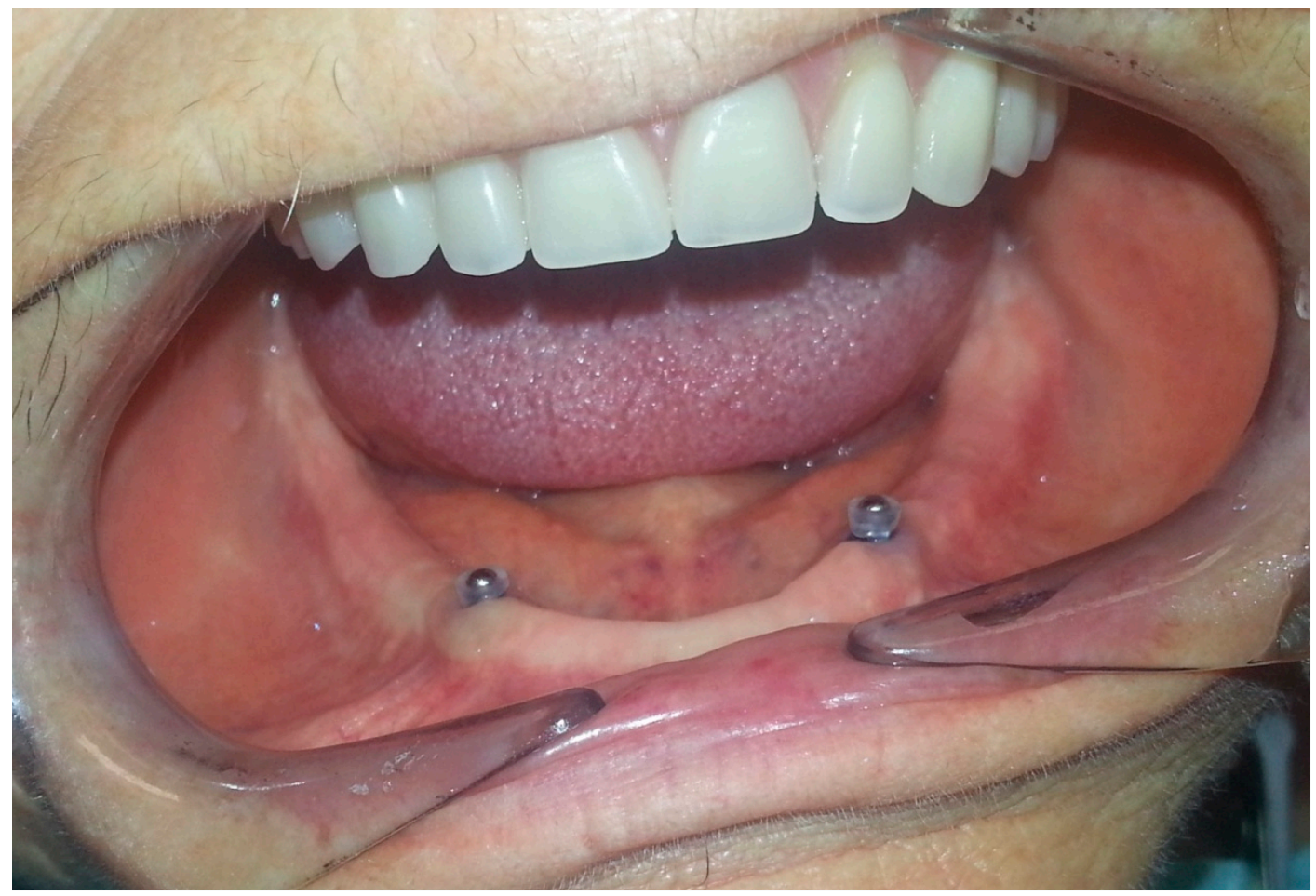

Figure 4. Silicone tube cut to the right height, positioned intra-orally trying not to cover the entire ball attachment.

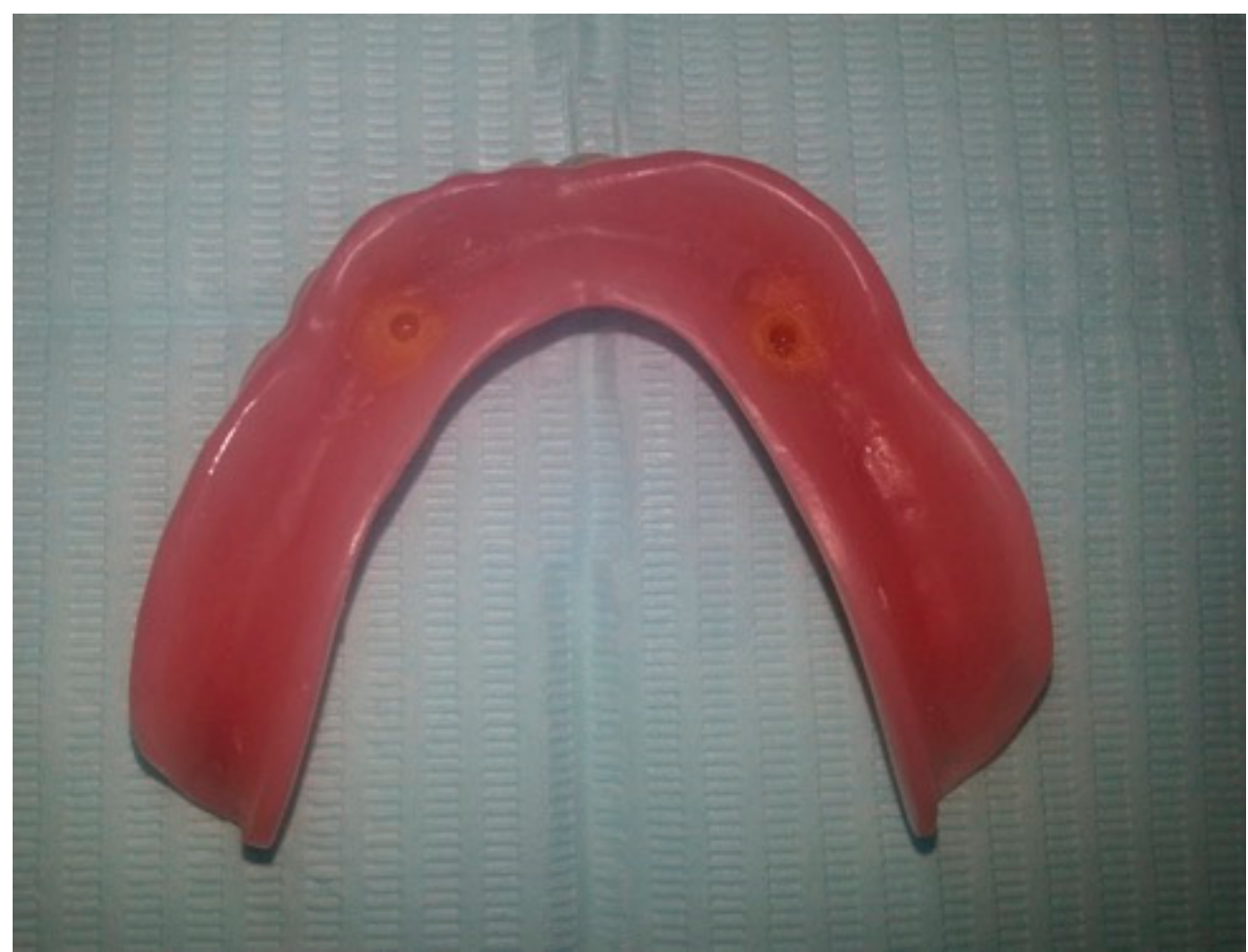

Figure 5. Lower mobile prosthesis with inserted Flexacril soft resin to increase the retention and adaptability of the prosthesis. 


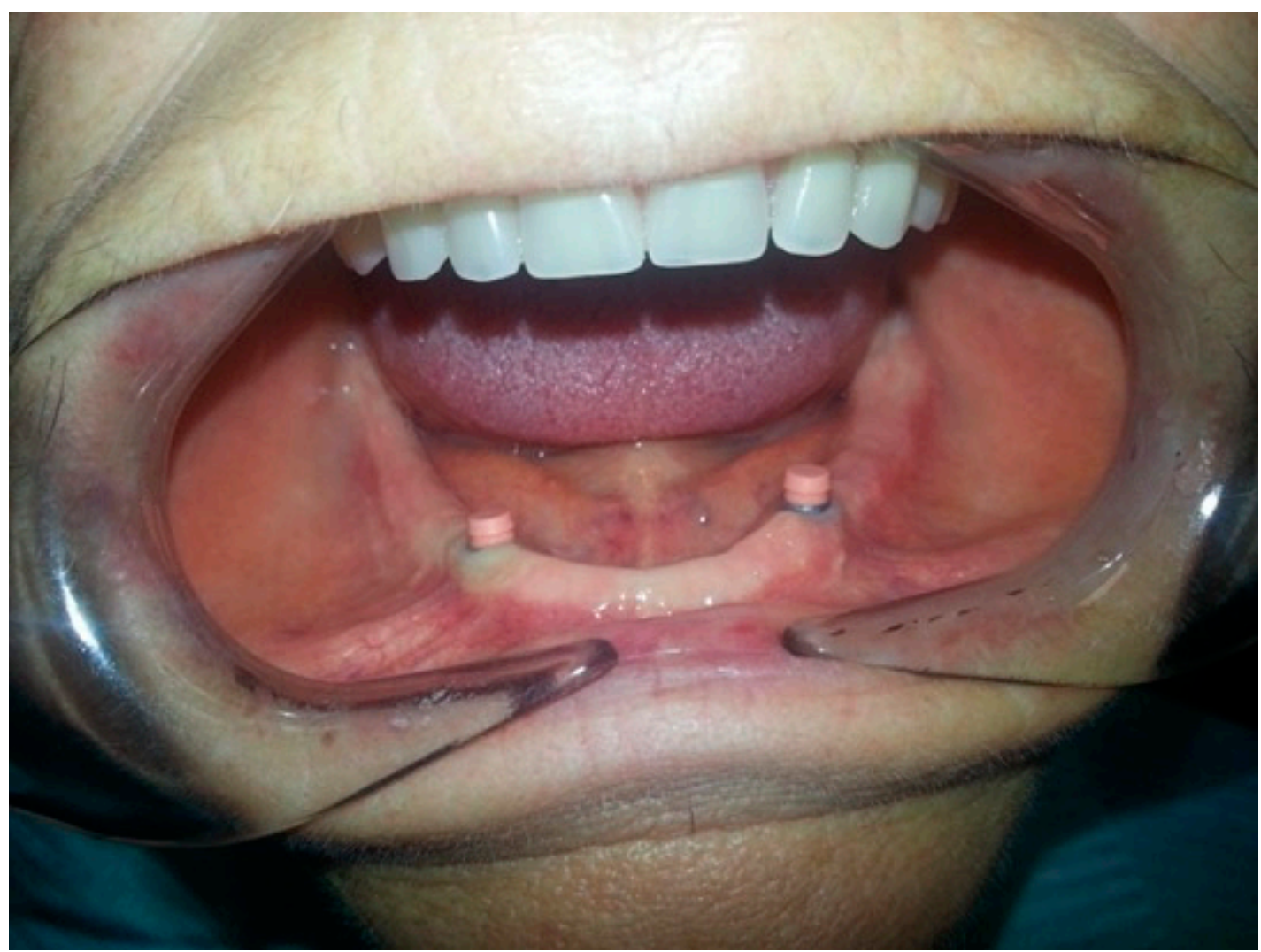

Figure 6. Teflon inserted on ball attachment.

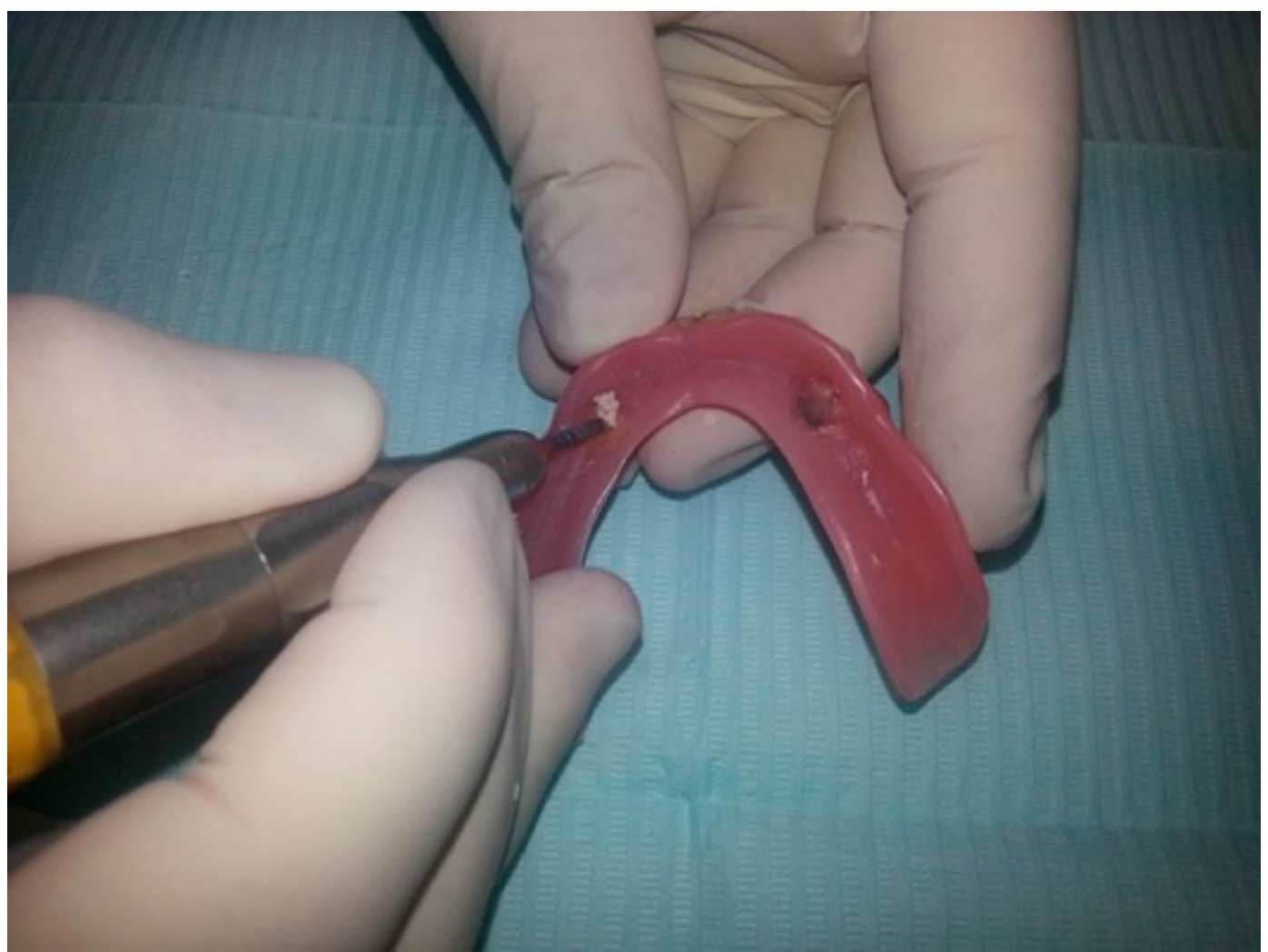

Figure 7. Soft resin removal. 


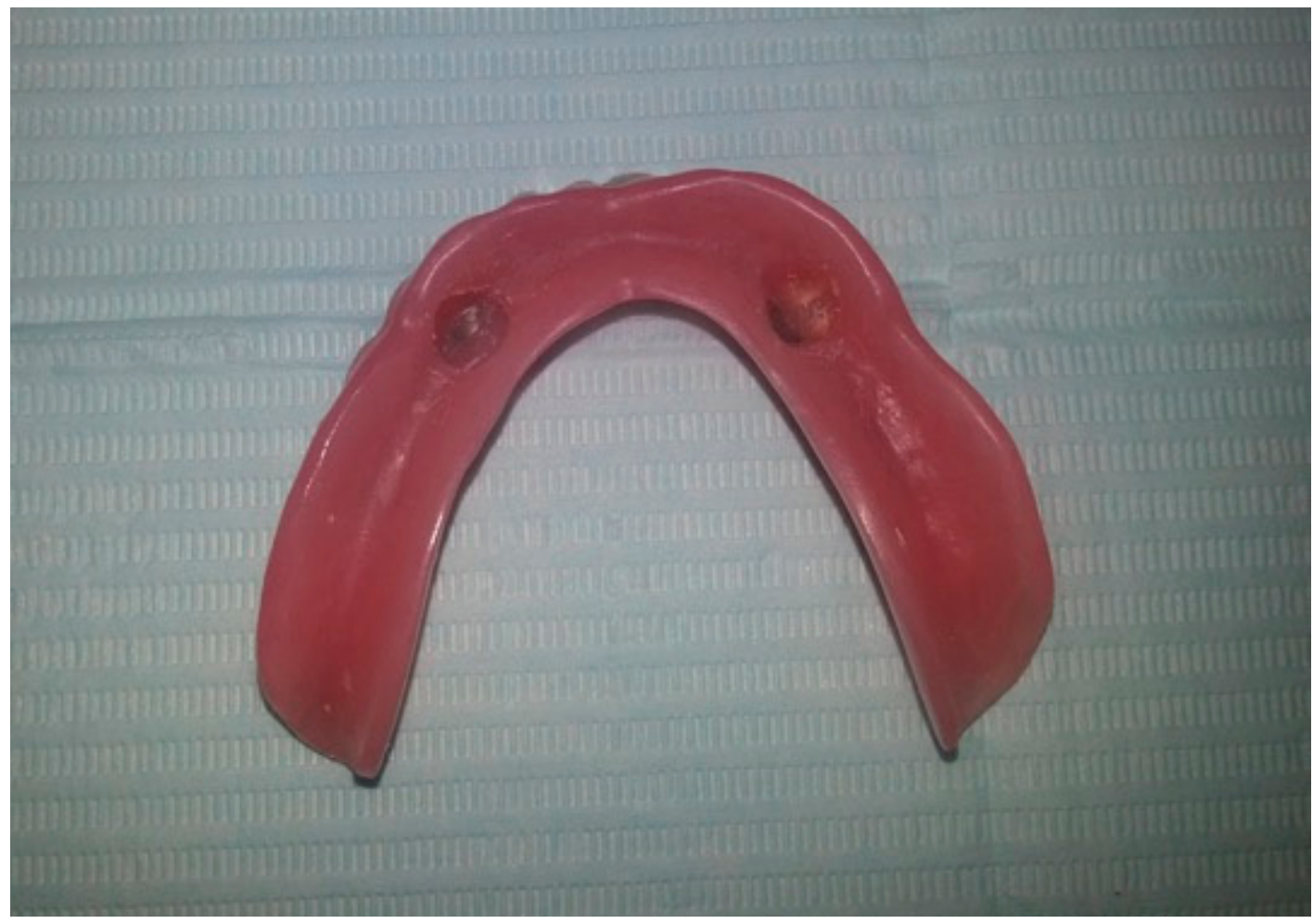

Figure 8. Prosthesis ready for the insertion of the hard resin.

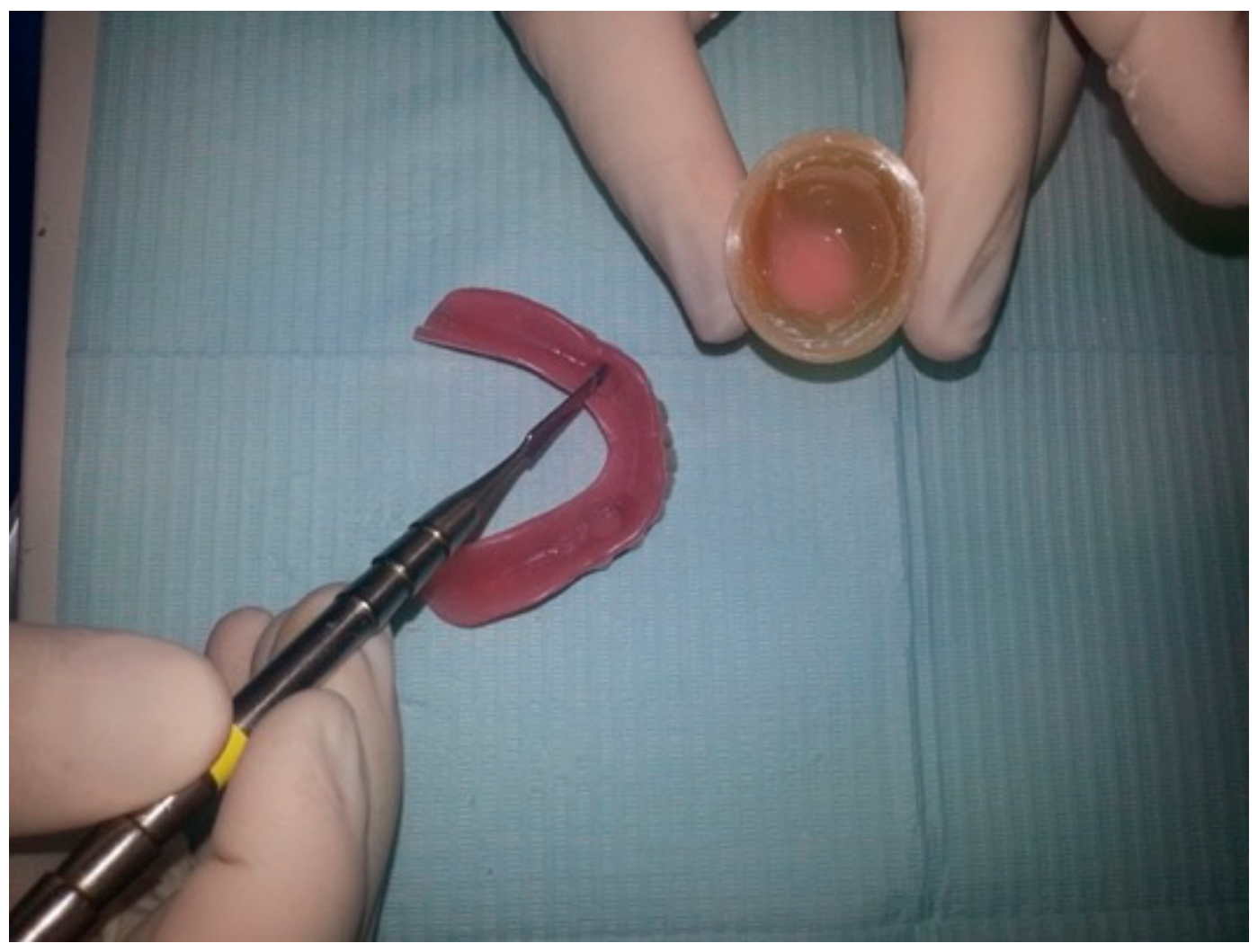

Figure 9. Insertion of Paladur self-curing acrylic resin trying to fill the cavities for $50 \%$. 


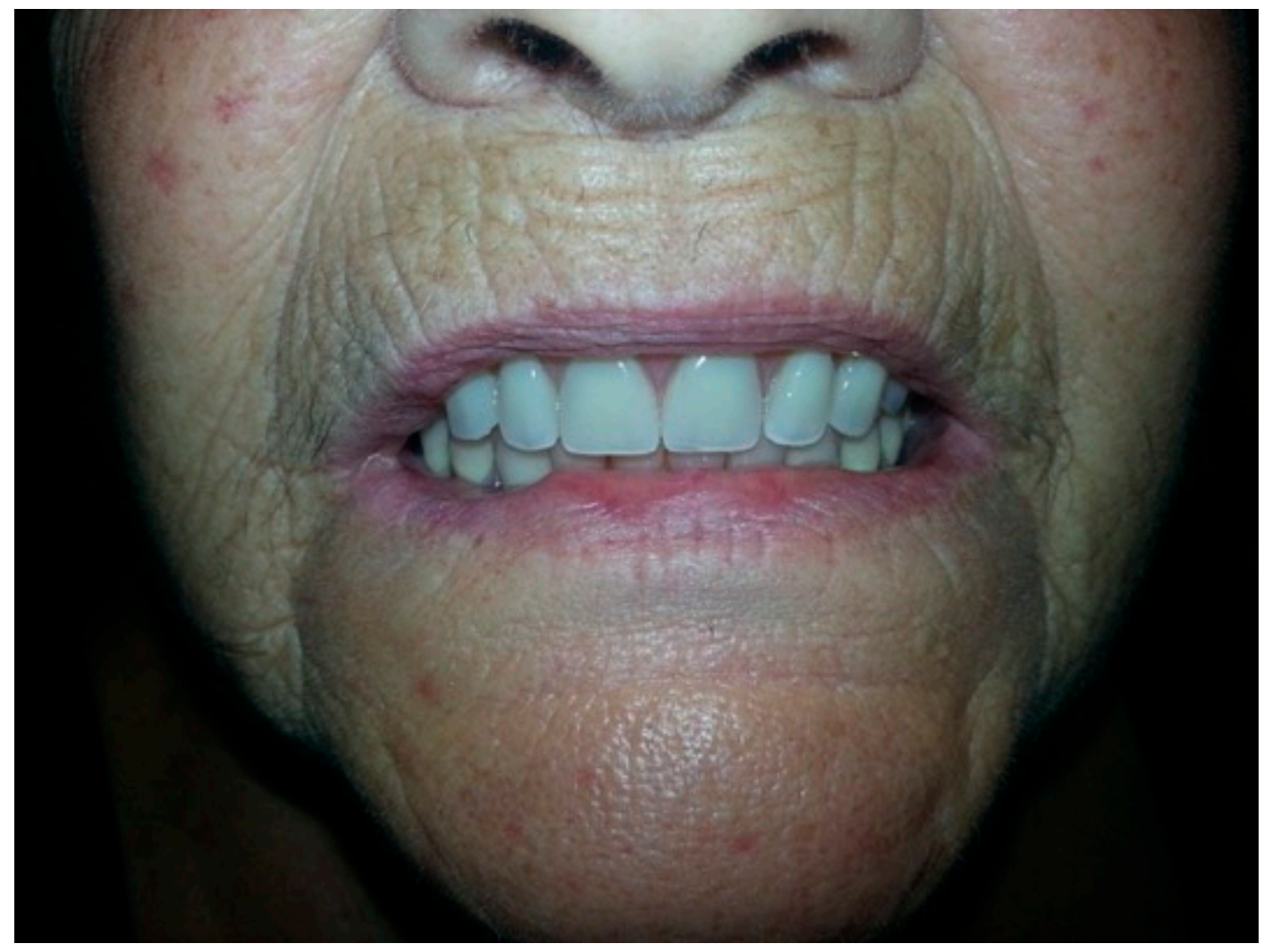

Figure 10. Prosthesis inserted into the oral cavity.

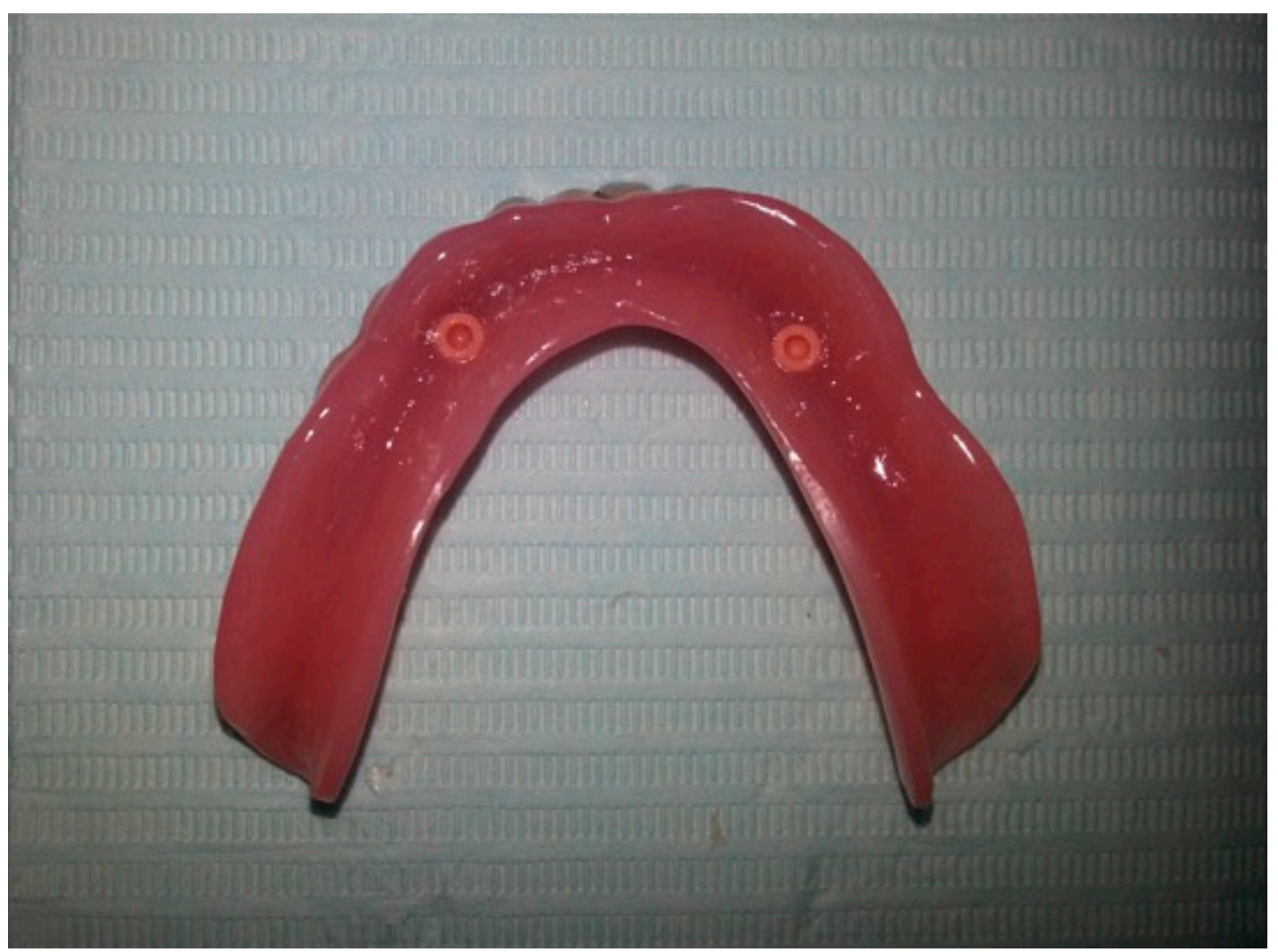

Figure 11. Prosthesis just removed after retentive fixation. 


\section{Results}

The use of this technique greatly helps the dentist as it reduces the risk, thanks to the silicone tube, that the resin may end up in the undercut of the ball-attachment, as well as reduces the working and finishing time as the amount of excess resin is very little (Figures 12 and 13). This technique does not stress the patient and the operators who collaborate throughout the operation. None of the treated patients required the use of anesthesia for the procedure, none showed signs nor symptoms of discomfort or pain during the treatment, and no post-op analgesic nor anti-inflammatory medication was administered. In each of the 13 patients, the procedure allowed the replacement of the Teflon o-ring with the prosthesis removal in absolute tranquility, with subsequent finishing with the portable micromotor and redelivery of the reconditioned product without having placed the Health Care Residence patient in dedicated dental facilities.

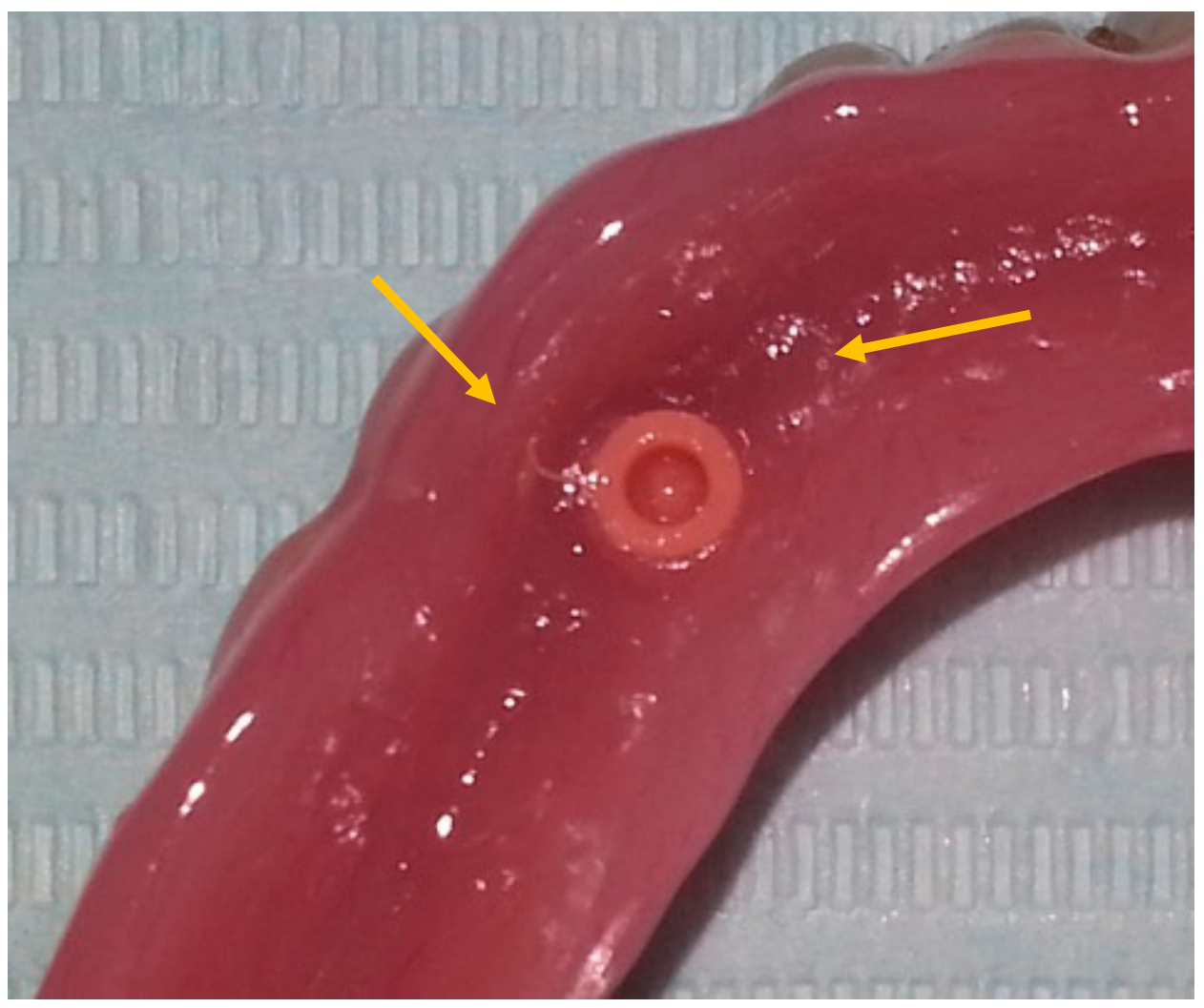

Figure 12. Detail of the slight deburring of the excess self-curing resin on the right side. 


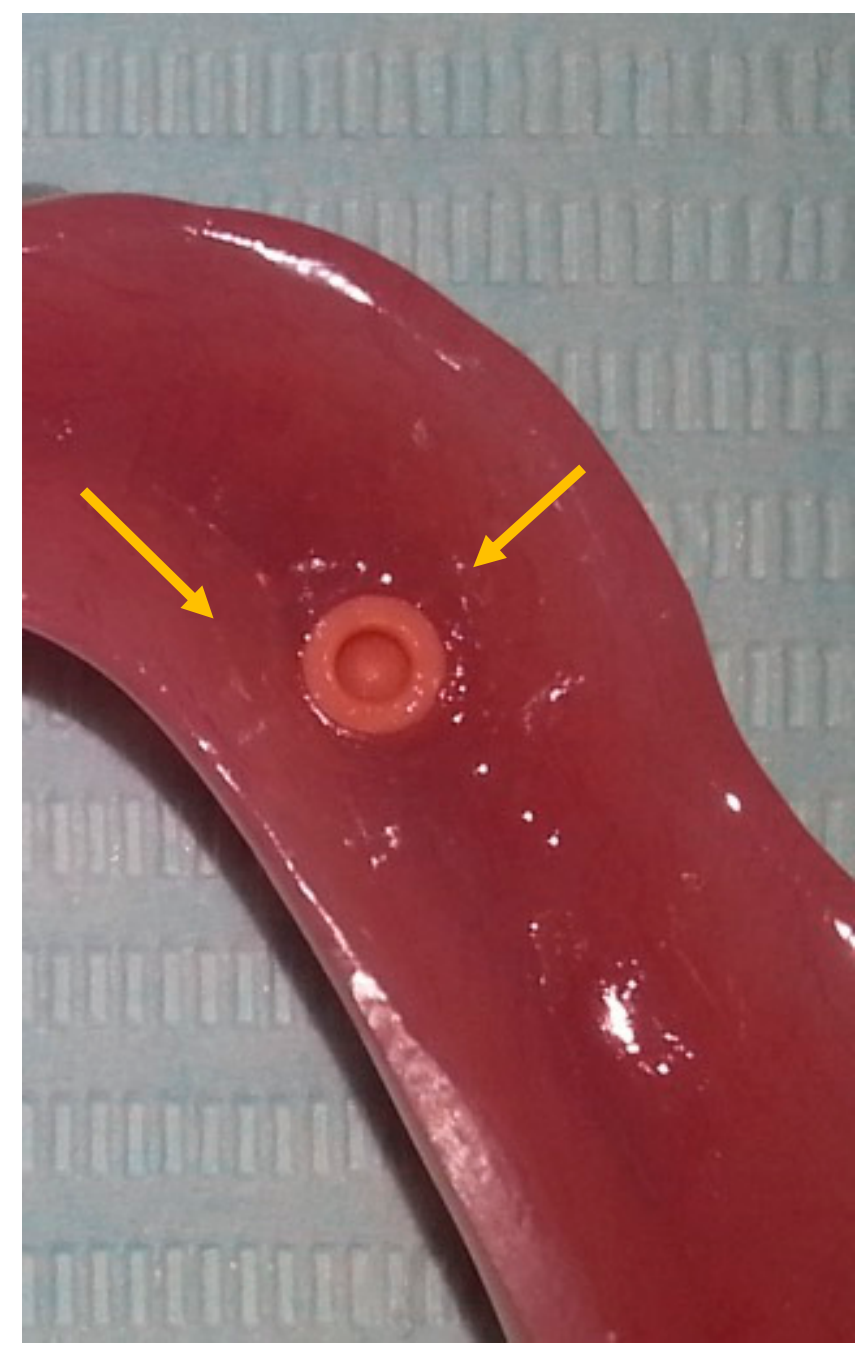

Figure 13. Detail of the slight deburring of the excess self-curing resin on the left side.

\section{Conclusions}

This technique allows us to stabilize the prosthesis in elderly patients who do not cooperate or are in bed even when the space is reduced due to the insertion of the metal container for Teflon. The use of the silicone tube allowed the dentist to perform a safe and comfortable procedure for patients, both in terms of Teflon insertion and for the duration of the operating session This technique differs from that used for locator and equator attacks [12,13] in that the latter use a standard-sized flat ring as a thickness that, once inserted, rests directly on the base of the locator or equator. In the OTK, (ball attachment) the distance between the base and the retention ball is greater. The silicone ring cannot be used for ball bindings because there is a risk that the resin will end up under the retry ball by not allowing the removal of the prosthesis. This inconvenience cannot occur with the silicone tube because it does not have a standard size, but it is the doctor who cuts the silicone tube according to the height of the OTK, i.e., according to the protrusion/distance of the ball attachment from the gum.

Author Contributions: Conceptualization, S.C.; methodology, A.L.; resources, A.B.; data curation, S.L.; validation, M.B.; writing - original draft preparation, S.C.; writing-review and editing, D.L. supervision, G.C. All authors have read and agreed to the published version of the manuscript.

Funding: This research received no external funding.

Conflicts of Interest: The authors declare no conflict of interest. 


\section{References}

1. Volkert, D. Nutrition in dementia. Internist 2017, 58, 141-148. [CrossRef] [PubMed]

2. Parrotta, I.; Maltais, M.; Rolland, Y.; Spampinato, D.A.; Robert, P.; de Souto Barreto, P.; Vellas, B. The association between apathy and frailty in older adults: A new investigation using data from the Mapt study. Aging Ment. Health 2019, 14, 1-5. [CrossRef] [PubMed]

3. Bell, C.L.; Lopez, R.P.; Mahendra, N.; Tamai, A.; Davis, J.; Amella, E.J.; Masaki, K. Person-centered Feeding Care: A Protocol to Re-introduce OralFeeding for Nursing Home Patients with Tube Feeding. J. Nutr. Health Aging 2016, 20, 621-627. [CrossRef] [PubMed]

4. Okamoto, N.; Amano, N.; Nakamura, T.; Yanagi, M. Relationship between tooth loss, low masticatory ability, and nutritional indices in the elderly: A cross-sectional study. BMC Oral Health 2019, 19, 110. [CrossRef] [PubMed]

5. Caccianiga, G.; Rey, G.; Baldoni, M.; Paiusco, A. Clinical, Radiographic and Microbiological Evaluation of High Level Laser Therapy, a New Photodynamic Therapy Protocol, in Peri-Implantitis Treatment; A Pilot Experience. Biomed. Res. Int. 2016. [CrossRef] [PubMed]

6. Caccianiga, G.; Rey, G.; Fumagalli, T.; Cambini, A.; Denotti, G.; Giacomello, M. Photodynamic therapy (association diode laser/hydrogen peroxide): Evaluation of bactericidal effects on periodontopathic bacteria: An in vitro study. Eur. J. Inflamm. 2012, 10 (Suppl. 2), 101-106. [CrossRef]

7. Caccianiga, G.; Cambini, A.; Rey, G.; Paiusco, A.; Fumagalli, T.; Giacomello, M. The use of laser diodes superpulses in implantology. Eur. J. inflamm. 2012, 10, 97-100. [CrossRef]

8. Čelebić, A.; Peršić, S.; Kovačić, I.; Buković, D.; Lešić, N.; Rener-Sitar, K. Comparison of Three Prosthodontic Treatment Modalities for Patients with Periodontally Compromised Anterior Mandibular Teeth: A 2-year follow-up study. Acta Stomatol. Croat. 2019, 53, 4-16. [CrossRef] [PubMed]

9. Karabuda, C.; Yaltirik, M.; Bayraktar, M. A clinical comparison of prosthetic complications of implant-supported overdentures with different attachment systems. Implant Dent. 2008, 17, 74-81. [CrossRef] [PubMed]

10. Abou-Ayash, S.; Enkling, N.; Srinivasan, M.; Haueter, M.; Worni, A.; Schimmel, M. Evolution of in vivo assessed retention forces in one-piece mini dental implant-retained mandibular overdentures: 5-Year follow-up of a prospective clinical trial. Clin. Implant Dent. Relat. Res. 2019, 16. [CrossRef] [PubMed]

11. Ceraulo, S. Modifica della dimensione vertical. Caso clinic. PROtech 2008, 9, 17-22, ISSN 15910067.

12. Ortensi, L.; Ortensi, M.; Minghelli, A.; Grande, F. Implant-Supported Prosthetic Therapy of an Edentulous Patient: Clinical and Technical Aspects. Prosthesis 2020, 2, 140-152. [CrossRef]

13. Tallarico, M.; Cervino, G.; Scrascia, R.; Uccioli, U.; Lumbau, A.; Meloni, S.M. Minimally Invasive Treatment of Edentulous Maxillae with Overdenture Fully Supported by a Cad/Cam Titanium Bar with a Low-Profile Attachment Screwed on Four or Six Implants: A Case Series. Prosthesis 2020, 2, 53-64. [CrossRef] 